\title{
SỰ HỘI TỤ CƯA PHƯƠNG PHÁP BIẾN THỂ NGHIÊMM DÀN HỒI TRONG LÝ THUYẾT QUÁ TRÌNH BIẾN DẠNG ĐÀN DẺO
}

\author{
VŨ KHǺ BẢY
}

Phương pháp biến thể nghiệm đàn hồi trong lý thuyết quá trình biến dạng đàn dẻo được trình bày trong [1], nhưng cho đển nay chưa có một chứng minh nào về sự hội tự của nó cả về lý thuyết cũng như phương pháp số. Trong bài này khằng định sự hội tụ cưa phương pháp biến thể nghiệm đàn hồi băng phương pháp số thông qua việc giải hai bài toán đàn dẻo, đồng thời khảo sát tốc độ hội tụ trong cả quá trình đàn dẻo.

\section{TRẠG THÁI ĐÀN DẺO CỦA ỐNG DÀY CHỊU ÁP LỰC TRONG VÀ LỰC KÉO DỌC TRỤC}

Xét ống dày, dài, bán kính trong bằng $a$, bán kính ngoài bằng $b$, chịu áp lực đều ớ trong $P(t)$ và lực kéo dọc trục $Q(t)$. Ống làm bằng vật liệu tái bền, không nén được $(\nu=0,5)$, không lực khối.

Ta xét bài toán trong hệ tọa độ trụ. Do điều kiện đới xứng và ống dài vô hạn nên các biến dạng và ứng suất là hàm của $r$ và $t$ :

$$
\begin{aligned}
& E_{z z}=e_{z z} \equiv e_{z z}(t), \quad E_{r r}=e_{r r} \equiv r_{r r}(r, t), \\
& E_{\theta \theta}=e_{\theta \theta} \equiv e_{\theta \theta}(r, t), \quad E_{r \theta}=E_{r z}=E_{\theta z}=0 .
\end{aligned}
$$

Các thành phần ứng suất, biến dạng phải thỏa mãn các phương trình và các điều kiện biên:

$$
\begin{gathered}
\frac{\partial \sigma_{r r}}{\partial r}+\frac{\sigma_{r r}-\sigma_{\theta \theta}}{r}=0, \quad e_{r r}=\frac{\partial u_{r}}{\partial r} ; \quad e_{\theta \theta}=\frac{u_{r}}{r} \\
\sigma_{r r}= \begin{cases}-P(t) & \text { khi } r=a \\
0 & \text { khi } r=b,\end{cases} \\
Q(t)=2 \pi \int_{a}^{b} \sigma_{z z} r d r, \quad S_{i j}=2 G e_{i j}+\int_{0}^{t} R_{i j k \ell} d e_{k \ell} .
\end{gathered}
$$

Trong đó

$$
R_{i j k \ell}=-2 G W_{1} \delta_{i k} \delta_{j \ell}+3 G\left(W_{1}-W_{2}\right) \frac{S_{i j} S_{k \ell}}{\sigma_{u}^{2}}
$$


Si $W_{1}=1-\frac{\sigma_{u} k(s)}{3 G}, W_{2}=1-\frac{\phi^{\prime}(s)}{3 G}, \phi^{\prime}(s)$ và $k(s)$ là các hàm đặc trưng cho tính chất cula vật ?àu. Ở đây do vật liệu tái bền tuyến tính nên: $\phi^{\prime}(s)=E^{\prime}$ khi $s \geq s_{0}, \phi^{\prime}(s)=E$ khi $s<s_{0}$ và $(s)=1 / s$.

Chia quá trình đặt tải từ thời điểm ban đầu đến thời điểm đang xét thành n giai đoạn, khi ó ta có:

$$
S_{i j}^{(n)}=2 G e_{i j}^{(n)}+\sum_{m=1}^{n} \int_{t_{m-1}}^{t_{m}} R_{i j k \ell} \dot{e}_{k \ell} d t=2 G e_{i j}^{(n)}+\Delta_{i j}^{(n)}
$$

'ới ước lượng tích phân:

$$
\int_{t_{m-1}}^{t_{m}} R_{i j k \ell} \dot{e}_{k \ell} d t=\frac{1}{2}\left[R_{i j k \ell}^{(m-1)}+R_{i j k \ell}^{(m)}\right] \Delta e_{k \ell}^{(m)}=\Delta_{m} e_{i j}
$$

Thuật giải ơ đây là biết nghiệm ơ cuối giai đoạn $n-1$ (tức là sễ biết được các $\Delta_{m} e_{i j}$ ) ta iác định nghiệm cưa bài toán ở giai đoạn thứ $n$. Trong giai đoạn này ta giải gần đúng liên tiếp. Jghiệm gần đúng thứ $k$ cần thởa mãn:

$$
\begin{gathered}
\frac{\partial \sigma_{r r}^{\langle n, k\rangle}}{\partial r}+\frac{\sigma_{r r}^{\langle n, k\rangle}-\sigma_{\theta \theta}^{\langle n, k\rangle}}{r}=0 \\
e_{r r}^{\langle n, k\rangle}=\frac{\partial u_{r}^{\langle n, k\rangle}}{\partial r}, \quad e_{\theta \theta}^{\langle n, k\rangle}=\frac{u_{r}^{\langle n, k\rangle}}{r} \\
S_{i j}^{(n, k)}=2 G e_{i j}^{(n, k)}+\sum_{m=1}^{n-1} \Delta_{m} e_{i j}+\Delta_{n}^{(k)} e_{i j}
\end{gathered}
$$

với các điều kiện biên:

$$
\begin{gathered}
\sigma_{r r}=\left\{\begin{array}{lll}
-P\left(t_{n}\right) & \text { với } & r=a \\
0 & \text { với } & r=b
\end{array}\right. \\
Q\left(t_{n}\right)=2 \pi \int_{a}^{b} \sigma_{z z}^{(n . k)} r d r
\end{gathered}
$$

Trong đó:

$$
\Delta_{r_{i}}^{(k-1)} e_{i j}=\frac{1}{2}\left[R_{i j k \ell}^{(n-1)}+R_{i j k \ell}^{(n, k-1)}\right]\left[e_{k \ell}^{(n, k-1)}-e_{k \ell}^{(n-1)}\right]
$$

và

$$
R_{i j k \ell}^{(n, k-1)}=-2 G W_{1}^{(n, k-1)} \delta_{i k} \delta_{j \ell}+3 G\left(W_{1}^{(n, k-1)}-W_{2}^{(n, k-1)}\right) \frac{S_{i j}^{(n, k-1)} \cdot S_{k \ell}^{(n, k-1)}}{\left[\sigma_{u}^{(n, k-1)}\right]^{2}}
$$

̛̛̉ gần đúng thứ $k=0$ lấy $\Delta_{n}^{(0)} e_{i j}=0$.

Đặt

$$
\sum_{m=1}^{n-1} \Delta_{m} e_{r r}+\Delta_{n}^{(k)} e_{r r}=R^{(n, k)} ; \quad \sum_{m=1}^{n-1} \Delta_{m} e_{\theta \theta}+\Delta_{n}^{(k)} e_{\theta \theta}=B^{(n, k)}
$$

Tìr điều kiện $e_{r r}+e_{\theta \theta}+e_{z z}=0$ nên từ (1.2) ta được:

$$
\frac{\partial u_{r}}{\partial r}+\frac{u_{r}}{r}=-e_{z z} \Rightarrow u_{r}=-e_{z z} \frac{r}{2}+\frac{C}{r} \Rightarrow e_{r r}=-\frac{e_{z z}}{2}-\frac{C}{r^{2}}
$$


và

$$
e_{\theta \theta}=-\frac{e_{z z}}{2}+\frac{C}{r^{2}} ; \quad C \text { là hăng số. }
$$

Thay vào (1.3) ta được:

$$
\begin{aligned}
& S_{r r}^{\langle n, k\rangle}=\sigma_{r r}^{\langle n, k\rangle}-\sigma^{\langle n, k\rangle}=-G\left[e_{z z}^{\langle n, k\rangle}+\frac{2 C^{\langle n, k\rangle}}{r^{2}}\right]+R^{\langle n, k\rangle} \\
& S_{\theta \theta}^{\langle n, k\rangle}=\sigma_{\theta \theta}^{\langle n, k\rangle}-\sigma^{\langle n, k\rangle}=G\left[-e_{z z}^{\langle n, k\rangle}+\frac{2 C^{\langle n, k\rangle}}{r^{2}}\right]+B^{\langle n, k\rangle} \\
& S_{z z}^{\langle n, k\rangle}=\sigma_{z z}^{\langle n, k\rangle}-\sigma^{\langle n, k\rangle}=2 G e_{z z}^{\langle n, k\rangle}-R^{\langle n, k\rangle}-B^{\langle n, k\rangle}
\end{aligned}
$$

Đem thay vào phương trình cân bằng (1.1) ta dược:

$$
\frac{\partial \sigma_{r r}^{\langle n, k\rangle}}{\partial r}=\frac{4 G C^{\langle n, k\rangle}}{r^{3}}+\frac{B^{\langle n, k\rangle}-R^{\langle n, k\rangle}}{r} \Rightarrow \sigma_{r r}^{\langle n, k\rangle}=\int_{a}^{r} \frac{B^{\langle n, k\rangle}-R^{\langle n, k\rangle}}{r} d r-\frac{2 G C^{\langle n, k\rangle}}{r^{2}}+C_{1}^{\langle n, k\rangle}
$$

Xác định $C^{(n, k)}$ và $C_{1}^{(n, k)}$ từ điều kiện biên $(1.4 \mathrm{a})$ :

$$
\begin{aligned}
C^{\langle n, k\rangle} & =\left\{P\left(t_{n}\right)-\int_{a}^{b} \frac{B^{\langle n, k\rangle}-R^{\langle n, k\rangle}}{r} d r\right\} \frac{a^{2} b^{2}}{2 G\left\langle b^{2}-a^{2}\right\rangle} \\
C_{1}^{\langle n, k\rangle} & =2 G C^{\langle n, k\rangle} \frac{1}{a^{2}}-P\left(t_{n}\right)
\end{aligned}
$$

Thay vào ta được:

$$
\begin{aligned}
& \sigma_{r r}^{\langle n, k\rangle}=2 G C^{\langle n, k\rangle}\left(\frac{1}{a^{2}}-\frac{1}{r^{2}}\right)+\int_{a}^{r} \frac{B^{\langle n, k\rangle}-R^{\langle n, k\rangle}}{r} d r-P\left(t_{n}\right) \\
& \sigma_{\theta \theta}^{\langle n, k\rangle}=\sigma_{r r}^{\langle n, k\rangle}+\frac{4 G C^{\langle n, k\rangle}}{r^{2}}+\dot{B}^{\langle n, k\rangle}-R^{\langle n, k\rangle}
\end{aligned}
$$

Tùr điều kiện biên (1.4b) ta được:

$$
\begin{aligned}
& Q\left(t_{n}\right)=\pi \int_{a}^{b}\left\{\sigma_{r r}^{\langle n, k\rangle}-\sigma_{\theta \theta}^{\langle n, k\rangle}-3\left(R^{\langle n, k\rangle}+B^{\langle n, k\rangle}\right)\right\} r d r+6 G \pi \int_{a}^{b} e_{z z}^{\langle n, k\rangle} r d r \\
& \Rightarrow e_{z z}^{\langle n, k\rangle}=\frac{1}{3 G \pi\left\langle b^{2}-a^{2}\right\rangle}\left\{Q\left(t_{n}\right)+\pi \int_{a}^{b}\left[3\left(R^{\langle n, k\rangle}+B^{\langle n, k\rangle}\right)-\sigma_{r r}^{\langle n, k\rangle}-\sigma_{\theta \theta}^{\langle n, k\rangle}\right] r d r\right\}
\end{aligned}
$$

Do vậy ta được:

$$
\sigma_{z z}^{(n . k)}=\frac{1}{2}\left\{6 G e_{z z}^{(n, k)}-3 R^{(n, k)}-3 B^{(n, k)}-\sigma_{r r}^{(n, k)}-\sigma_{\theta \theta}^{(n, k)}\right\} .
$$

Tìr đó ta tính được: $e_{\theta \theta}^{(n, k)}, e_{z z}^{(n, k)}, S_{r r}^{(n, k)}, S_{\theta \theta}^{(n, k)}, S_{z z}^{(n, k)}$ và

$$
\sigma_{u}^{(n, k)}=\sqrt{\frac{3}{2} S_{i j}^{(n, k)} S_{i j}^{(n, k)}}=\sqrt{\frac{3}{2}\left\{\left[S_{r r}^{(n, k)}\right]^{2}+\left[S_{\theta \theta}^{(n, k)}\right]^{2}+\left[S_{r r}^{(n, k)}+S_{\theta \theta}^{(n, k)}\right]^{2}\right\}}
$$


Do $S^{(n)}=S^{(n-1)}+S_{n}$ nên $S^{(n, k)}=S^{(n-1)}+S_{n}^{(k)}$ trong đó

$$
\begin{aligned}
& S^{(n)}=\sqrt{\frac{2}{3}} \int_{0}^{t_{n}} \sqrt{\dot{e}_{i j} \dot{e}_{i j}} d t=\sqrt{\frac{2}{3}} \sum_{m=1}^{n} \int_{i_{m-1}}^{t_{m}} \sqrt{e_{i j} \dot{e}_{i j}} d t \\
& S_{n}^{(k}=\sqrt{\frac{2}{3}} \sqrt{\left(e_{i j}^{(n, k)}-e_{i j}^{(n-1)}\right) \cdot\left(e_{i j}^{(n, k)}-e_{i j}^{(n-1)}\right)}
\end{aligned}
$$

Gọi $\varphi$ là góc lệch giữa véctơ ứng suất và quỹ đạo biến dạng tạ có:

$$
\cos \varphi=\frac{S_{i j} \dot{e}_{i j}}{\sigma_{u} \cdot V_{u}} \quad \text { vậy } \quad(\cos \varphi)^{(n, k)}=\frac{S_{i j}^{(n, k)} \cdot\left(e_{i j}^{(n, k)}-e_{i j}^{\left(r_{u}-1\right)}\right)}{\sigma_{u}^{(n, k)} S_{n}^{(k)}}
$$

Biểu đồ 1. Đồ thị quỹ đạo tái ngoài kéo nén ống trụ dày.

(1) - cách đặt tải 1

(2) - cách đặt tải 2

(3) - cách đặt tải 3
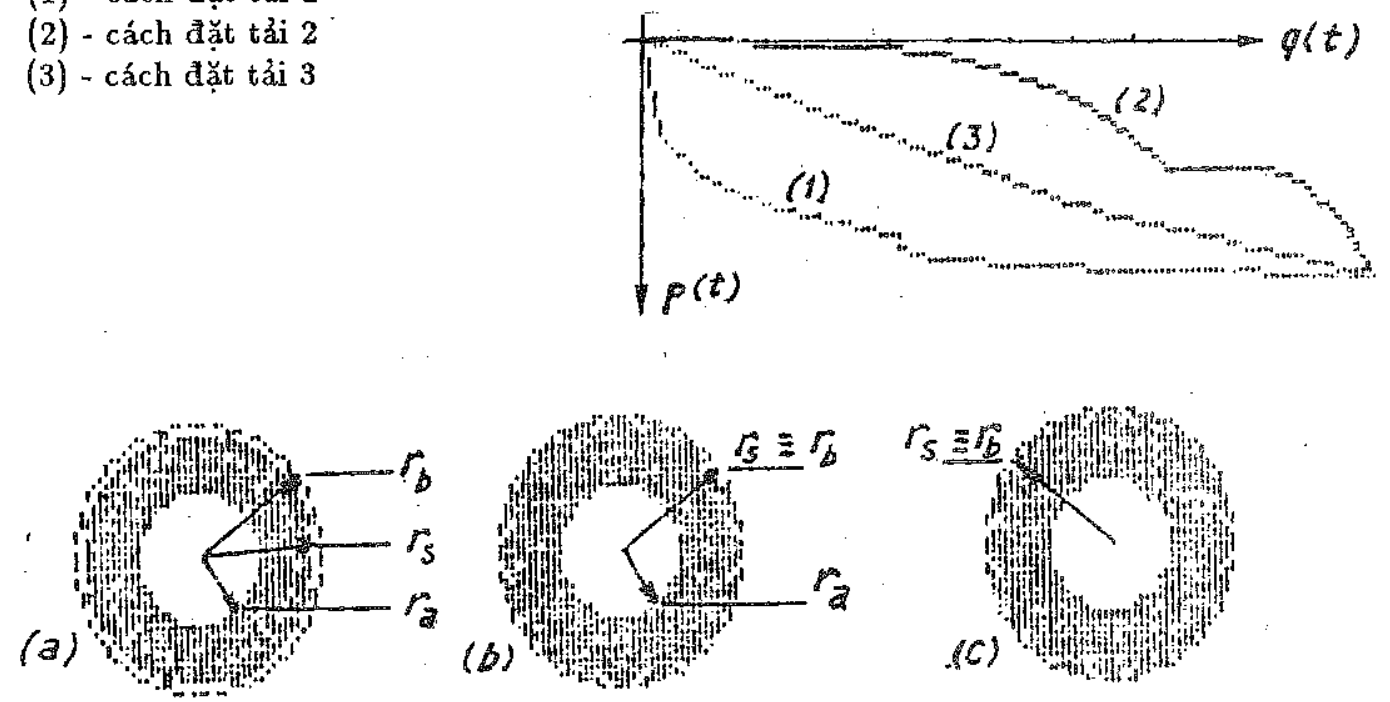

Hỉnh 1. Hình ảnh đàn déo ơ một số giai đoạn trong cách đặt tăii 1.

(a) Giai đoạn $22: p=0,50651, q=0,98433$

(b) Giai đoạn $23: p=0,50917, q=1,0523$

(c) Giai đoạn $24: p=0,5117, q=1,1242$

Ở mồi bước ta cần kiển tra giá trị của $(\cos \varphi)^{(n, k)}$. Nếu:

$(\cos \varphi)^{(n, k)} \geq 0$ - ứng với quá trình đặt tái.

$(\cos \varphi)^{(n, k)}<0$ - ứng với quá trình cất tải.

Vì các đại lượng là hàm của $r$ và $t$ nên ta chia bán kính từ $r_{a}$ đến $r_{b}$ thành $m$ đoạn bởi $m+1$ điểm chia. Đưa vào các đại lượng không thứ nguyện:

$$
q(t)=\frac{Q(t)}{\pi\left(a^{2}-b^{2}\right) \sigma_{s}}, \quad p(t)=\frac{P(t)}{\sigma_{s}}, \quad \bar{S}_{i j}=\frac{S_{i j}}{\sigma_{s}}, \quad h=\frac{r_{a}}{r_{b}}, \quad \gamma=\frac{1-h^{2}}{3+h^{4}}
$$

Áp dụng tính toán với thép $40 \mathrm{X}$; có $\frac{E^{\prime}}{E}=0,028, \sigma_{s}=858 \frac{M N}{m^{2}}, G=70000 \frac{M N}{m^{2}}$ với $h=0 ; 5$, $m=20, n=25$; đặt tải theo 3 cách và với các cách chịa giai đoạn theo tham sổ tải khác rhhau. Bảng 1 ghi kết quả tính toán $b 3$ giai đoạn theo cách đặt tải 1 . Sai số giữa hai lần lặp thứ 3 và 
thứ 2 của các đại lượng (tại các điểm có tọa ạ̣̉̂̂ $r=r_{0}$ ) ớ tất cả các giai đoạn khắc đều nhỏ hơn ở 3 giai đoạn này.

Kết quáa tính toán theo 3 cách đặt tải (nhu trong biểu đồ 1) cho thấy răng: saì số giữa hai lần lặp thứ 3 và thứ 2 trong suốt quá trình của các đại lượng ứng suất đều nhỏ hơn $4,3 \%$, của các đại lượng biến dạng đều nhỏ hơn $3 \%$, của cường độ ứng-suẩt đều nhỏ kơn $2 \%$; sai số giữa hai lần lặp thứ 8 và thứ 7 ờ mọi giai đoạn tải của các đại lượng ứng suất đều nhỏ hơn $3 \%$, cử các đại lượng biên dạng đều nhỏ hơn $2,4 \%$, cưa cường độ ứng suất đều nhỏ hơn $1,5 \%$.

\section{Bảng 1}

\begin{tabular}{|c|c|c|c|c|c|c|c|c|}
\hline Tai & $\begin{array}{c}\text { Số } \\
\text { lần lặp }\end{array}$ & $\frac{\sigma_{\theta \theta}}{\sigma_{s}}$ & $\frac{\sigma_{z z}}{\sigma_{s}}$ & $\frac{\sigma_{u}}{\sigma_{s}}$ & $e_{r r} \cdot 10^{3}$ & $e_{\theta \theta} \cdot 10^{3}$ & $e_{z=\pi} \cdot 10^{3}$ & $\cos \varphi$ \\
\hline \multirow[t]{2}{*}{ Giai đoạn 22} & 2 & 0,52052 & 0,57975 & 1,0579 & $-6,64$ & 3,06 & 3,579 & 0,8622 \\
\hline & 3 & 0,50336 & 0,55578 & 1,0371 & $-6,72$ & 3,12 & 3,602 & 0,9218 \\
\hline \multirow[t]{2}{*}{$p=050651$} & saì sơ & $3,4 \%$ & $4,3 \%$ & $2 \%$ & $1,2 \%$ & $1,86 \%$ & $6,3 \cdot 10^{-3}$ & $6,5 \%$ \\
\hline & 7 & 0,49253 & 0,54497 & 1,0263 & $-6,93$ & 3,23 & 3,702 & 0,9693 \\
\hline \multirow[t]{2}{*}{$q=0,98433$} & 8 & 0,49627 & 0,54293 & 1,0269 & $-6,96$ & 3,26 & 3,704 & 0,9764 \\
\hline & sai sớ & $7,3.10^{-3}$ & $3,7.10^{-3}$ & $5,7.10^{-4}$ & $4,4.10^{-3}$ & $8,8,10^{-3}$ & $5,4.10^{-4}$ & $7,3.10^{-3}$ \\
\hline \multirow[t]{2}{*}{ Giai đoạn 23} & 2 & 0,52696 & 0,63020 & 1,0914 & $-7,38$ & 3,21 & 4,169 & 0,8562 \\
\hline & 3 & 0,53511 & 0,64067 & 1,1009 & $-7,60$ & 3,31 & 4,354 & 0,9303 \\
\hline \multirow[t]{2}{*}{$p=0,50917$} & sai số & $1,5 \%$ & $1,6 \%$ & $8,5.10^{-3}$ & $3,7 \%$ & $3 \%$ & $4,24 \%$ & $7,9 \%$ \\
\hline & 7 & 0,52201 & 0,62523 & 1,0865 & $-8,58$ & 3,70 & 4,878 & 0,9848 \\
\hline \multirow{2}{*}{$q=1,0253$} & 8 & 0,52273 & 0,62357 & 1,0858 & $-8,77$ & 3,79 & 4,983 & 0,9880 \\
\hline & sai số & $1,4.10^{-3}$ & $2,7,10^{-3}$ & $5,9.10^{-4}$ & $2,2.10 \%$ & $2,3 \%$ & $2,1 \%$ & $3,2.10^{-3}$ \\
\hline \multirow[t]{2}{*}{ Giai doạn 24} & 2 & 0,54973 & 0,71559 & 1,1534 & $-9,20$ & 3,69 & 5,493 & 0,8384 \\
\hline & 3 & 0,55750 & 0,72401 & 1,1615 & $-9,48$ & 3,77 & 5,719 & 0,9119 \\
\hline \multirow[t]{2}{*}{$p=0,51176$} & sai số & $1,4 \%$ & $1,2 \%$ & $7.10^{-3}$ & $3 \%$ & $2,1 \%$ & $3,7 \%$ & $8 \%$ \\
\hline & 7 & 0,56078 & 0,73235 & 1,1678 & $-10,65$ & 4,10 & 6,479 & 0,9801 \\
\hline \multirow[t]{2}{*}{$q=1,1242$} & 8 & 0,56044 & 0,73227 & 1,1682 & $-10,93$ & 4,28 & 6,658 & 0,9842 \\
\hline & sai số & $6.10^{-4}$ & $1,2.10^{-4}$ & $3,6.10^{-4}$ & $2,5 \%$ & $2,3 \%$ & $2,6 \%$ & $4.10^{-3}$ \\
\hline
\end{tabular}

\section{TRANG THÁI DÀN DẺO CỨ THANH TRÒN CHUU XOÁ̉ VÀ LỤC KÉO DỌC TRỤC}

Xét thanh tròn, đài, bán kính bằng $a$, làm băng vật liệa không nér đạ̛̛̣c, tái bền tuyến tính, không lực khổi chịu lực kéo dọc trực $P(t)$ và mômen xoắn $M(t)$.

Đới với bài toán này ta vẫn dừng già thiết bán kính thẳng và thiết diện phẳng. Ở đây vẫn xét bài toán trong hệ tọa độ trụ. Do tính chất của lực ngoài tác dụng nện ta thấy rằng các đại lượng biểu thị ứng suất và biến dạng không phụ thuộc vào góc $\theta$ và $z$, các đụi lượng biểu thị về chuyển dịch không phụ thuộc vào góc $\theta$. Các thành phần ứng suất chị còn lại thành phần $\sigma_{z z}$ và $\sigma_{\theta z}$ là khác không còn các thành phần khác đều bằng không, do vậy các phrương trinh cân bàng đều thóa mãn. 
Gọi $\beta$ là góc quay giữa hai thiết diện cách nhau một đơn vị, khi dó do già thiết thiết diện phẳng và bán kính thằng chuyển dịch $u_{\theta}=r . z$, do vậy $u_{\theta z}=\frac{1}{2} \beta r$, và do già thiết thiết diện phẳng nền $e_{z z}=e_{z z}(t)$. Do $\theta_{e}=0$ nên:

$$
\frac{\partial u_{r}}{\partial r}+\frac{u_{r}}{r}=-e_{z z} \rightarrow u_{r}=-\frac{1}{2} e_{z z \cdot r}+\frac{C}{r} ; C \text { là hăng số. }
$$

Tì̀ điều kiện $r=0$ thì $u_{r} \doteq 0$ nên $C=0$, vậy $u_{2}=-\frac{1}{2} e_{z z} . r$, do đó ta có:

$$
e_{r \mathrm{r}}=-\frac{1}{2} e_{z z}(t), \quad e_{\theta \theta}=-\frac{1}{2} e_{z z}(t), \quad e_{\theta z}=\frac{1}{2} \beta(t) . r, \quad e_{z z}=e_{z z}(t)
$$

còn các đại lượng $e_{\mathrm{rz}}=e_{\mathrm{r} \theta}=0$.

Chia thời gian từ đầu đến thời điểm đang xêt thành $n$ đoạn, khi đó tại giai đoạn đang xét (thứ $n$ ) nghiệm của bài toán sẽ được giải bằng phương pháp xâp xậ liên tiếp trên cơ sở biết được nghiệm ơ cuối giai đọan trước (giai đoạn thứ $n-1$ ). Tại gần đúng thứ $k$ ớ giai đoạn $n$, các thành phàn ứng suất sẽ liên hệ với các thành phần biến dạng:

$$
S_{i j}^{(n, k)}=2 G e_{i j}^{(n, k)}+\sum_{m=1}^{n-1} \Delta_{m} e_{i j}+\Delta_{n}^{(k)} e_{i j}
$$

và các điều kiện biên:

$$
P\left(t_{r z}\right)=2 \pi \int_{0}^{a} \sigma_{z z}^{(n, k)} r d r, \quad M\left(t_{n}\right)=2 \pi \int_{0}^{a} S_{\theta z}^{(n, k)} r^{2} d r
$$

Trong đó:

với

$$
\Delta_{n}^{(k-1)} e_{i j}=\frac{1}{2}\left[R_{i j k \ell}^{(n-1)}+R_{i j k \ell}^{(n, k-1)}\right]\left[e_{k \ell}^{(n, k-1)} 1-e_{k \ell}^{(n-1)}\right]
$$

$$
R_{i j k \ell}^{(n, k-1)}=-2 G W_{1}^{(n, k-1)} \delta_{i k} \delta_{j \ell}+3 G\left(W_{1}^{(n, k-1)}-W_{2}^{(n, k-1)}\right) \frac{S_{i j}^{(n, k-1)} S_{k \ell}^{(n . k-1)}}{\left[\sigma_{u}^{(n, k-1)}\right]^{2}}
$$

và

$$
W_{2}=1-\frac{E^{\prime}}{E}, \quad W_{1}=1-\frac{\sigma_{u} \cdot k(s)}{E}, \quad \text { do } \quad k(s)=\frac{1}{s} \text { nên } W_{1}=1-\frac{o_{u}}{\sigma_{s}+E \cdot s_{1}}
$$

Trong đó $s_{1}$ là độ dài cung biến dạng băt đầu sau giai đọan đàn hồi đên thời điểm đang xét. Ờ gần đúng thứ $k=0$ ta lấy $\Delta_{n}^{(0)} e_{i j}=0$.

Do $\sigma_{r r}=\sigma_{\theta \theta}=0$ nên tù̀ (2.1) kết hợp với (2.2) ta luôn có $\sum_{m} \Delta_{m} e_{r r}=\sum_{m} \Delta_{m} e_{\theta \theta}$ br mọi giai đoạn. Ký hiệu:

$$
\begin{aligned}
& \sum_{m=1}^{n-1} \Delta_{m} e_{r r}+\Delta_{n}^{(k)} e_{r r}=\sum_{m=1}^{n-1} \Delta_{m} e_{\theta \theta}+\Delta_{n}^{(k)} e_{\theta \theta}=R^{(n-1)}+\Delta_{n}^{(k)} e_{r r}=R^{(n, k)} \\
& \sum_{m=1}^{n-1} \Delta_{m} e_{\theta z}+\Delta_{n}^{(k)} e_{\theta z}=F^{(n-1)}+\Delta_{n}^{(k)} e_{\theta z}=F^{(n, k)}
\end{aligned}
$$

Khi đó từ (2.2) ta có: 


$$
\begin{aligned}
& S_{r r}^{(n, k)}=S_{\theta \theta}^{(n, k)}=-\sigma^{(n, k)}=-G e_{z z}^{(n, k)}+R^{(n, k)}=-\frac{1}{3} \sigma_{z z}^{(n, k)}, \\
& S_{z z}^{(n, k)}=\sigma_{z z}^{(n, k)}-\sigma^{(n, k)}=2 G e_{z z}^{(n, k)}-2 R^{(n, k)}, \\
& S_{\theta z}^{(n, k)}=2 G e_{\theta z}^{(n, k)}+F^{(n, k)} .
\end{aligned}
$$

Thay vào điều kiện biên ta được:

$$
P\left(t_{n}\right)=2 \pi \int_{0}^{a} 3\left(G e_{z z}^{(n, k)}-R^{(n, k)} r d r\right.
$$

Do vậy:

$$
e_{z z}^{(n, k)}=\frac{1}{3 \pi G a^{2}}\left\{P\left(t_{n}\right)+6 \pi \int_{0}^{a} R^{(n, k)} r d r\right\}
$$

Do

$$
e_{\theta z}=\frac{1}{2} \beta^{(n, k)} \cdot r \Rightarrow S_{\theta z}^{(n, k)}=G . r . \beta^{(n, k)}+F^{(n, k)}
$$

Từ điều kiện biên thứ 2 của (2.3) thay vào ta có:

$$
M\left(t_{n}\right)=2 G \pi \int_{0}^{a} \beta^{(n, k)} \cdot r^{3} d r+2 \pi \int_{0}^{a} F^{(n, k)} \cdot r^{2} d r
$$

suy ra

$$
\beta^{(n, k)}=\frac{1}{\pi G a^{4}}\left[2 M\left(t_{n}\right)-4 \pi \int_{0}^{a} F^{(n, k)} \cdot r^{2} d r\right]
$$

Có

$$
S^{(n, k)}=S^{(n-1)}+S_{n}^{(k)} \quad \text { với } \quad S_{n}^{(k)}=\sqrt{\frac{2}{3}} \int_{t_{n-1}}^{t_{n}} \sqrt{\dot{\dot{e}_{i j} \dot{e}_{i j}}} d t
$$

thay vào ta được:

$$
S_{n}^{(k)}=\sqrt{\left[e_{z z}^{(n, k)}-e_{z z}^{(n-1)}\right]^{2}+\frac{4}{3}\left[e_{\theta z}^{(n, k)}-e_{\theta z}^{(n-1)}\right]^{2}}
$$

và

$$
\sigma_{u}^{(n, k)}=\sqrt{\left[\sigma_{z z}^{(n, k)}\right]^{2}+3\left[S_{\theta z}^{(n, k)}\right]^{2}}
$$

Gọi $\varphi$ là góc lệch giữa véctơ ứng suất và quỹ đạo biến dạng ta có:

$$
(\cos \varphi)^{(n, k)}=\frac{S_{i j}^{(n, k)} \cdot\left(e_{i j}^{(n, k)}-e_{i j}^{(n-1)}\right)}{\sigma_{u}^{(n, k)} \cdot S_{n}^{(k)}}
$$

Đura vào các đại lượng không thứ nguyên:

$$
q(t)=\frac{2 M(t)}{a^{3} \pi \sigma_{s}} ; \quad p(t)=\frac{P(t)}{3 \pi a^{2} \cdot \sigma_{s}}, \quad \alpha=a \cdot \beta, \quad \bar{S}_{i j}=\frac{S_{i j}}{\sigma_{s}}
$$


Biể dồ 2. Dồ thị tăi ñgoài kéo xoån trụ tròn.

(1) - cách a ăt tai 1

(2) - cách dạt tải 2

(3) - cách đặt tải 3
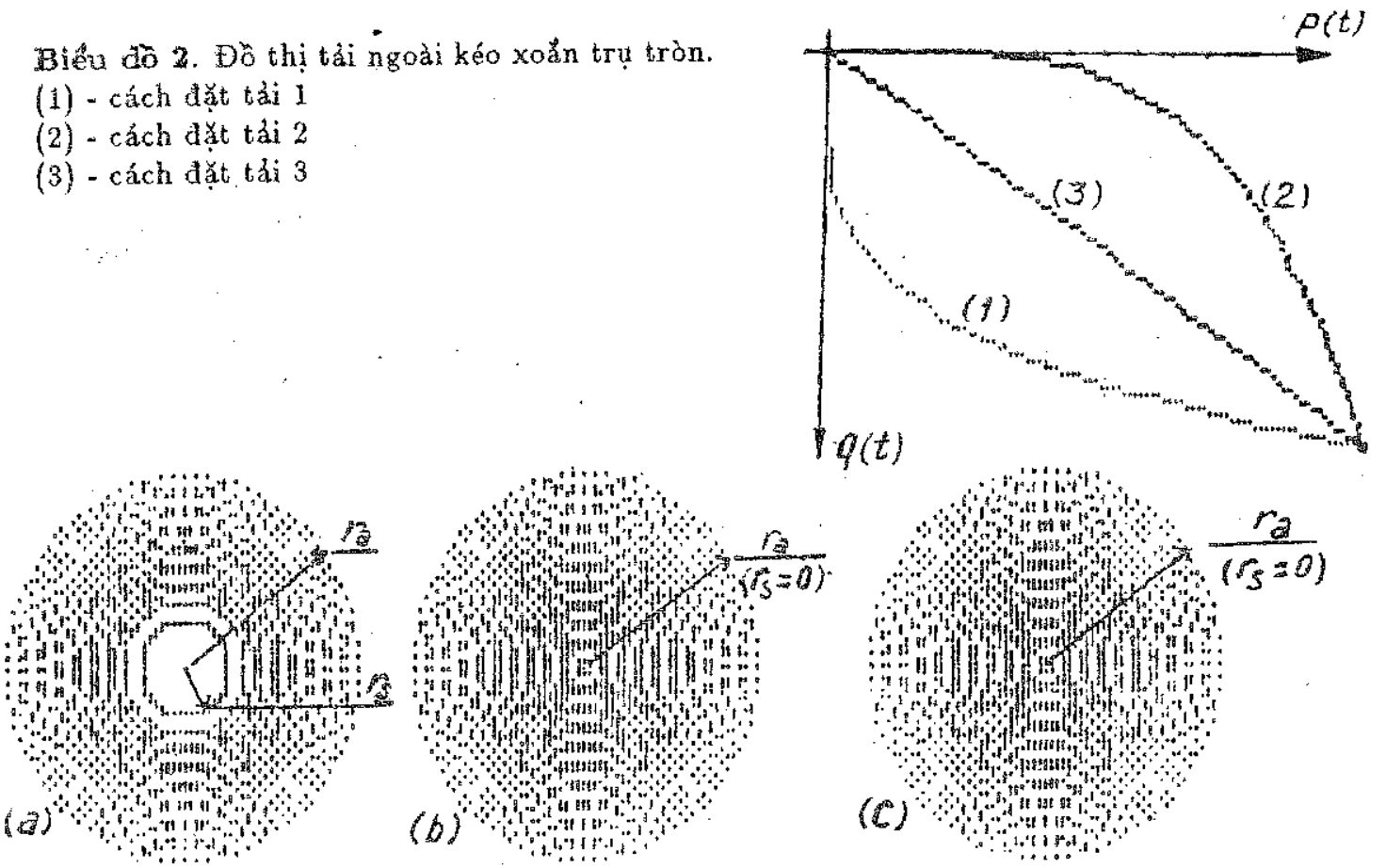

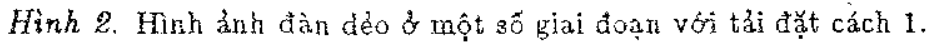

(a) - Giai doan 36: $q=0,82159 ; p=0,27000$

(b) Giai doạn 39: $q=0,85512 ; q=0,31687$

(c) Giai doạn 40: $q=0,86604 ; p=0,3333$

Áp dụng tính toán với thép $40 \mathrm{X}$ nên $\frac{E^{\prime}}{E}=0,028, \sigma_{s}=858 \frac{M N}{m^{2}}, G=70000 \frac{M N}{m^{2}}$. Chia đều các giai đoạ theo tham số tải $t$ (tăng tì 0 đén 40 ) \&ै 3 cách ăăt tải:

Dặt tải cách 1: $q(t)=\frac{\sqrt{3}}{2} \sqrt{\frac{t}{n}}, p(t)=\frac{1}{3}\left(\frac{t}{n}\right)^{2}$

Dặt tải cách 2: $q(t)=\frac{\sqrt{3}}{2}\left(\frac{t}{n}\right)^{2}, \frac{1}{3} \sqrt{\frac{t}{n}}$

Đăt tải cách $3: q(t)=\frac{\sqrt{3}}{2} \frac{t}{n}, \frac{1}{3} \frac{t}{n}$

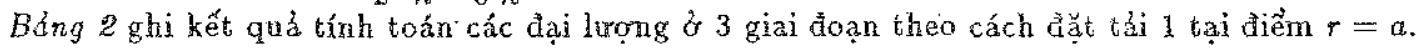

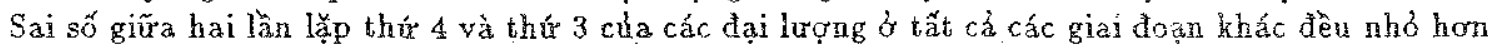
לे giai đỏñ này.

Kết quá tính soán đởi với cả 3 cách đặt tái cho thấy rằng: Sai số giữa hai lần lặp thứ 4 và 3

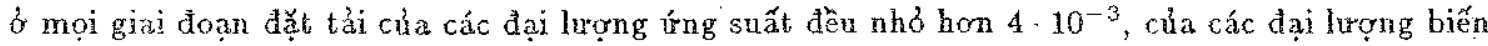
dang đều nhó hơn $3 \cdot 10^{-3}$, cúa cường dộ ưng suất đều nhó hơn $10^{-3}$, của cos $\varphi$ đều nhó hon

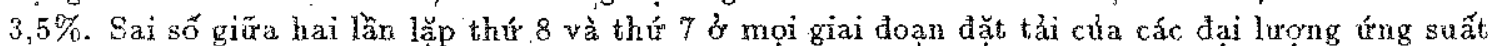
đều nhỏ hơn $10^{-5}$, cha crà̀ng dộ úng 3 uất và của các đại lượng biến dạng dều nhó hom $10^{-4}$, cha $\cos \varphi$ đều nhỏ hơn hoăc ơ múrc $10^{-3}$.

Nhân xét: Qua việc giải hai bài toán trên ta nhận thấy:

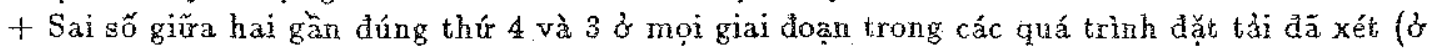
cả hai bài toán) cula các đại lượng ứng suất dều nhỏ hơn Â\%, của các đại lượng biến dạng đều nhỏ hơn $3 \%$, cula cường độ ứng suất đều nhỏ hơn $2 \%$.

+ Tốc độ hội tự trong các cách đặt tải của bài toán I chậm hơn tốc độ hội tụ trong các cách đặt tầi ça bài toán II, lý do vì các cách đặt tải trong bài toán I phức tap hơn.

+ Do lấy điều kiện $\sigma_{u} \geq \sigma$, ta áp dụng quy luật dèo, $\sigma_{u}<\sigma_{s}$ ta áp dụng quy luật đàn hồi

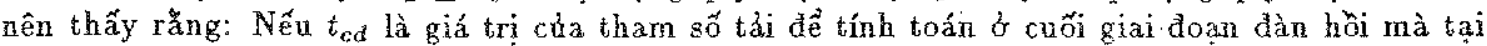


đây $\sigma_{u}$ sai khác $\sigma_{s}$ khá lớn thì trong khoảng từ $t_{c d}$ đến $t_{c d+1}$ đáng lễ ta áp dụng quy luật đàn hồi nhưng từ lần lặp thứ 2 trớ đi ta đã áp dụng quy luật dẻo. Do vậy trong cách chia $n$ giai đoạn để tính toán ta cần chú ý đến điểm này vì nó ánh hường đến tốc độ hội tụ khá nhiều.

Bding 2

\begin{tabular}{|c|c|c|c|c|c|c|c|}
\hline Tai & $\begin{array}{c}\text { Số } \\
\text { lần lặp }\end{array}$ & $\frac{\sigma_{z z}^{\prime}}{\sigma_{s}}$ & $\frac{\tau_{\theta_{z}}}{\sigma_{s}}$ & $\frac{\sigma_{u}}{\sigma_{s}}$ & $e_{z z} \cdot 10^{3}$ & a. $\beta \cdot 10^{3}$ & $\cos \varphi$ \\
\hline \multirow[t]{2}{*}{ Giai doạn 36} & 3 & 0,76999 & 0,84458 & 1,6531 & 3,860 & 9,210 & 0,5666 \\
\hline & 4 & 0,77188 & 0,84440 & 1,6538 & 3,870 & 9,208 & 0,5819 \\
\hline \multirow[t]{2}{*}{$q=0,82159$} & sai số & $2,44.10^{-3}$ & $1,94.10^{-4}$ & $3,8.10^{-4}$ & $2,7.10^{-3}$ & $4.10^{-4}$ & $1,5 \%$ \\
\hline & 7 & 0,77220 & 0,84434 & 1,6538 & 3,874 & 9,200 & 0,5540 \\
\hline \multirow[t]{2}{*}{$p=0,27000$} & 8 & 0,77240 & 0,84431 & 1,6538 & 3,875 & 9,200 & 0,5534 \\
\hline & sai số & $2,5.10^{-4}$ & $3,3.10^{-5}$ & $2,9.10^{-5}$ & $1,9.10^{-4}$ & $3,4.10^{-5}$ & $1,1.10^{-3}$ \\
\hline \multirow[t]{2}{*}{ Giai doạn 39} & 3 & 0,95310 & 0,87218 & 1,7862 & $4,9.17$ & 9,320 & 0,5931 \\
\hline & 4 & 0,95690 & $0,87.193$ & 1,7868 & 4,950 & 9,310 & 0,5809 \\
\hline \multirow{2}{*}{$q=0,85512$} & sai số & $1,9.10^{-3}$ & $2,8.10^{-4}$ & $3,4.10^{-4}$ & $3,6.10^{-3}$ & $8,6.10^{-4}$ & $2,1 \%$ \\
\hline & 7 & 0,95622 & 0,87176 & 1,7872 & 4,946 & 9,310 & 0,5745 \\
\hline \multirow{2}{*}{$p=0,31687$} & 8 & 0,95628 & 0,87175 & 1,7873 & 4,947 & 9,310 & 0,5742 \\
\hline & sai số & $6.10^{-5}$ & $9.10^{-6}$ & $10^{-5}$ & $1,1.10^{-4}$ & $2,3.10^{-5}$ & $5.10^{-4}$ \\
\hline \multirow[t]{2}{*}{ Giai doạn 40} & 3 & 1,0176 & 0,88109 & 1,8343 & 5,311 & 9,350 & 0,6077 \\
\hline & 4 & 1,0195 & 0,88084 & 1,8350 & 5,331 & 9,340 & 0,5953 \\
\hline \multirow[t]{2}{*}{$q=0,86604$} & sai số & $1,9.10^{-3}$ & $2,8.10^{-4}$ & $3,8.10^{-4}$ & $3,8 \cdot 10^{-3}$ & $9,3.10^{-4}$ & $2 \%$ \\
\hline & 7 & 1,0210 & 0,88065 & 1,8355 & 5,341 & 9,340 & 0,5883 \\
\hline \multirow[t]{2}{*}{$p=0,33333$} & 8 & 1,0211 & 0,88064 & 1,8355 & 5,346 & 9,340 & 0,5880 \\
\hline & sai số & $7,1.10^{-5}$ & $10^{-5}$ & $1,5.10^{-5}$ & $1,4.10^{-4}$ & $2,9.10^{-5}$ & $5,9.10^{-4}$ \\
\hline
\end{tabular}

\section{KẾT LUẬN}

Với những kết quả tính toán và các nhận xét trên ta rút ra các kết luận sau:

1- Phương pháp biến thể nghiệm đàn hồi thể hiện trên các bài toán được khăo sát có tính hội tụ ớ mọi giai đọan tải.

2 - Tốc độ hội tụ trong că quá trình phụ thuộc nhiêu vào mức độ phức tạp của quá trình đặt tải. Đối với các quá trình đặt tải đã xét, tốc độ hội tụ khá cao: Tại mọi giai đoạn tải, sai số giữa hai gần đúng thừ 4 và thứ 3 của các đại lượng ứng suất và biến dạng đều dưới $4 \%$; còn sai số giữa hai gàn đúng thứ 8 và 7 của chúng đều đã \& mức $10^{-3}$ hoăc $10^{-4}$.

3 -Với 4 lần lặp trên mồi giai đoạn tải (số giai đoạn từ 25 đến 35) thì thời gian tính toán cho mồi cách đặt tải trên máy vi tính AT mất khoảng từ 45 giây cho đến 60 giây.

Tác giả chần thành cạm ơn GS TS Đào Huy Bích đã hướng dẫn thực hiện công trình này,

Dia chi:

Trừng đại học Tông hợp Hà Nội

Nhận ngày 7/5/1992

\section{TÀI LIÊU THAM KHẢO}

1. Đào Huy Bích. Biến thể phương pháp nghiệm đàn hồi trong lý thuyết quá trình biến dạng đàn déo. Tạp chí khoa học Trường đại học Tồng hợp Hà Nội No 2, 1985.

(xem tiếp trang 26) 